\title{
Evaluating cabotegravir/rilpivirine long-acting, injectable in the treatment of HIV infection: emerging data and therapeutic potential
}

This article was published in the following Dove Press journal:

HIVIAIDS - Research and Palliative Care

\author{
Cristina Fernandez' \\ Clare $L$ van Halsema ${ }^{1,2}$ \\ 'Regional Infectious Diseases Unit, North \\ Manchester General Hospital, \\ Manchester, UK; ${ }^{2}$ Faculty of Education, \\ Liverpool School of Tropical Medicine, \\ Liverpool, UK
}

\begin{abstract}
Cabotegravir and rilpivirine long-acting injectable antiretroviral therapy for the treatment of HIV-1 infection brings promise of a new mode of delivery and potential solutions to some problems of oral therapy, but also new challenges and unanswered questions. Adding to the increasing body of evidence for newer two-drug combinations, phase II and phase III trial data to date demonstrate cabotegravir and rilpivirine combination injectable therapy to be non-inferior to selected oral triple-therapy alternatives. Most importantly, this therapy is reported to be acceptable to individuals taking the 4-weekly or 8weekly injections, despite frequent injection-site reactions. Key outstanding questions include management of missed or delayed dosing, drug interactions and management of virological failure, as well as the efficacy of cabotegravir and rilpivirine in all HIV-1 subtypes. We describe clinical evidence to date and efficacy and challenges in selected populations, including women; those with prior virological failure; individuals with a history of difficulty adhering to oral therapy and individuals with co-infections. We await real-world data and longer-term evidence while moving forward to this new era of antiretroviral therapy.
\end{abstract}

Keywords: antiretroviral therapy, integrase inhibitors, non-nucleoside reverse transcriptase inhibitors

\section{Introduction}

The global response to HIV (human immunodeficiency virus) has meant more people living with HIV (PLWH) are diagnosed, on treatment and living longer than ever before. ${ }^{1}$ But there still remain several unmet needs in HIV treatment across the globe, including more tolerable treatments, easier to adhere to, and, stemming from the UNAIDS 90-90-90 targets, ${ }^{2}$ addressing the "4th 90": improvement of quality of life in those living and aging with HIV. $^{3,4}$

Long-acting (LA) injectable therapy offers much-reduced dosing frequency and an additional option among two-drug combinations now available. Delivery of LA injectable antiretroviral therapy (ART) may require a conformational change in HIV healthcare delivery for some, with possibilities for more innovative, patientcentered health care models.

Pre-clinical data on LA cabotegravir and detail on rilpivirine pharmacokinetics have been described previously. ${ }^{5,6}$ Here, we describe phase II and III clinical trials, discuss resistance and suitability for specific populations.
Correspondence: Cristina Fernandez Regional Infectious Diseases Unit, North Manchester General Hospital, Delaunays

Road, Manchester M8 5RB, UK

Tel +44 I6I 7202733

Email cfernandez@doctors.org.uk 


\section{Drug characteristics}

Cabotegravir (GSK1265744) is an integrase strand transfer inhibitor (INSTI) of the carbamoyl pyridone class and a structural analog of dolutegravir. At the time of writing, cabotegravir is still an investigational antiretroviral agent, manufactured as a once-daily oral tablet and as an intramuscular (IM) and subcutaneous (SC) injectable. Not yet licensed, it is available, in Europe, through the manufacturer's expanded access program. ${ }^{7}$ Cabotegravir is formulated as an LA suspension for monthly to quarterly injection dosing. ${ }^{8}$ The half-life $\left(t_{1 / 2}\right)$ of LA injectable cabotegravir is $20-40$ days with some individuals having measurable concentrations cabotegravir up to a year after one single dose. ${ }^{8,9}$

Rilpivirine (TMC278) is a second-generation nonnucleoside reverse transcriptase inhibitor (NNRTI), licensed for the treatment of HIV-1 infection in individuals aged $\geq 12$ years with plasma HIV-1 RNA (ribonucleic acid) $\leq 100,000$ copies $/ \mathrm{mL}^{10,11}$ based on the ECHO and THRIVE studies. ${ }^{12,13}$ It is licensed as a $25 \mathrm{mg}$ oral tablet and in co-formulation as single tablet regimens with dual nucleoside reverse transcriptase inhibitors (NRTIs) and with dolutegravir. ${ }^{14-16}$ An LA injectable suspension has been developed for use with cabotegravir as injectable ART and, like cabotegravir, is available through an expanded access program. ${ }^{17}$ The $t_{1 / 2}$ of LA rilpivirine is 30-90 days. ${ }^{18-20}$

Oral rilpivirine needs to be taken with a meal for optimal absorption and cannot be co-administered with proton-pump inhibitors. ${ }^{21}$ Frequently occurring side effects (SE) are headaches, insomnia, dizziness and nausea, increases in total and low-density lipoprotein cholesterol, rises in pancreatic amylase and derangement of transaminases. ${ }^{22}$ Data on cabotegravir side effects to date come from trials and are discussed later.

Healthy volunteer studies of oral rilpivirine given with dolutegravir or cabotegravir demonstrate no significant pharmacokinetic interactions, no requirement to doseadjust either drug and a favorable short-term safety profile. $^{23}$ To date, there are two key phase II and two phase III clinical trials that support the efficacy and safety of oral and injectable cabotegravir with rilpivirine (CR); LATTE and LATTE-2, ATLAS and FLAIR.

\section{Clinical trial evidence Phase II studies}

The Long-Acting antireTroviral Treatment Enabling trial (LATTE) is a phase IIb randomized, partially blinded, dose-ranging study of once-daily oral $\mathrm{CR}$, which has now reported 96-week results. ${ }^{24}$ It compared three doseranging $\mathrm{CR}$ arms with two NRTIs plus efavirenz (Figure 1).

LATTE recruited ART-naïve HIV-1 positive adults (age $\geq 18$ years) with no major resistance-associated mutations (RAMs) at baseline. Investigators and participants were blinded only to the cabotegravir dose. As shown in Figure 1, at week 24, participants switched from the induction to maintenance phase. Participants in arms 1-3 only continued to the maintenance phase if HIV-1 RNA was $<50$ copies $/ \mathrm{mL}$, but those in arm 4 continued the same oral efavirenz-based regimen regardless of viral load (VL). Resistance testing was done at baseline and protocol-defined virological failure (PDVF). Randomization was stratified by baseline HIV-1 VL ( $<$ or $\geq 100,000$ copies/mL) and by NRTI backbone (abacavir-lamivudine or tenofovir-emtricitabine).

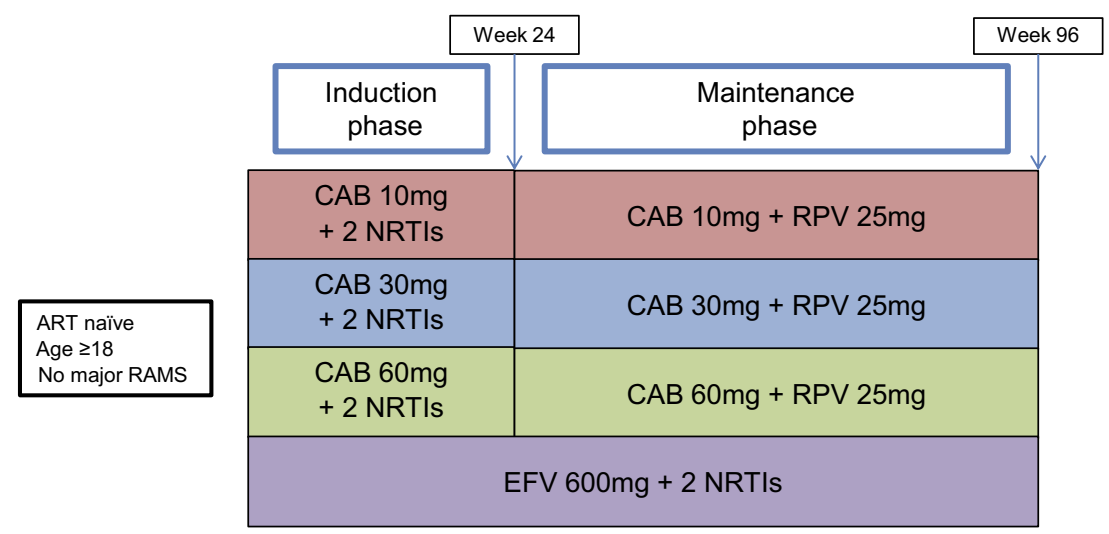

Figure I The Long-Acting antireTroviral Treatment Enabling trial (LATTE).

Abbreviations: ART, antiretroviral therapy; CAB, cabotegravir; RPV, rilpivirine; EFV, efavirenz; NRTis, nucleos(t)ide reverse transcriptase inhibitors; RAMS, resistance-associated mutations. 
The primary end point was an intention-to-treat (ITT) analysis in the exposed population of the proportion of participants with HIV-1 RNA $<50$ copies $/ \mathrm{mL}$ at week 48 . Of 243 patients enrolled and receiving at least one dose of an oral induction regimen, 207 patients took at least one dose of the oral maintenance regimen. At week 48, the combined overall response (HIV-1 VL $<50$ copies $/ \mathrm{mL}$ ) in the $\mathrm{CR}$ arms was $82 \%$ versus $71 \%$ in the 2 -NRTI and efavirenz arm. This difference in virological suppression was seen through to week 96 (combined $76 \%$ for CR vs $63 \%$ for 2-NRTI and efavirenz). In all CR regimens (regardless of cabotegravir dose), there were fewer participants with baseline HIV-RNA $\geq 100,000$ copies $/ \mathrm{mL}$ who sustained virological suppression at week 96 than with lower VLs. However, the authors concluded that numbers were too small to draw significant conclusions from this. An efficacy analysis of the ITT maintenance-exposed population found numerical superiority of cabotegravir $60 \mathrm{mg}$ and $30 \mathrm{mg}$ in achieving viral suppression at week 96 compared with $10 \mathrm{mg}$.

Overall, the CR regimens had better tolerability, fewer adverse event (AE)-related discontinuations and a lower proportion with virological non-response. In the cabotegravir arms, there was a higher frequency of headaches, depression and vomiting $(22 \%, 11 \%$ and $8 \%$ of the participants on cabotegravir versus $8 \%, 6 \%$ and $5 \%$ on efavirenz). Two participants taking cabotegravir $60 \mathrm{mg}$ had increasing transaminases on a background of steatohepatitis that improved on drug discontinuation. Dizziness, abnormal dreams, nausea, fatigue and insomnia were, as expected, the most frequent AEs in the NRTI and efavirenz arm. The
$30 \mathrm{mg}$ cabotegravir dose was taken forward for assessment as LA injectable.

LATTE-2 is a phase IIb, randomized, open-label, noninferiority study of LA CR, aiming to select a dosing regimen. ${ }^{25}$ It recruited ART-naïve HIV-1 positive adults (aged $\geq 18$ years) with CD4 counts $\geq 200$ cells $/ \mu \mathrm{L}$ and HIV$1 \mathrm{VL} \geq 1000$ copies $/ \mathrm{mL}$. As depicted in Figure 2, participants had a 20 -week oral induction phase of cabotegravir $30 \mathrm{mg}$ plus abacavir-lamivudine, with oral rilpivirine added at week -4 (16 weeks into induction phase). Those with HIV-1 RNA $<50$ copies $/ \mathrm{mL}$ at week -4 were randomized to one of the three maintenance treatment arms: LA cabotegravir $400 \mathrm{mg}$ plus rilpivirine $600 \mathrm{mg}$ every 4 weeks (q4w); LA cabotegravir $600 \mathrm{mg}$ plus rilpivirine $900 \mathrm{mg}$ every 8 weeks (q8w) or oral cabotegravir $30 \mathrm{mg}$ plus abacavir-lamivudine daily. The study allowed a 14day window for injections to more closely resemble realworld settings.

The primary outcomes were the proportion of the maintenance-exposed population with HIV-1 RNA $<50$ copies $/ \mathrm{mL}$ at week 32 of the maintenance period, proportion with PDVF and the incidence and severity of AEs and laboratory abnormalities. The extended analysis continued to 160 weeks, with those randomized to oral cabotegravir $30 \mathrm{mg}$ plus abacavir-lamivudine and virologically suppressed at 96 weeks switching to LA CR at 100 weeks, with optimized loading doses (cabotegravir $600 \mathrm{mg}$ and rilpivirine $900 \mathrm{mg}$ ) then $\mathrm{q} 4 \mathrm{w}$ or $\mathrm{q} 8 \mathrm{w}$ regimens. ${ }^{26}$

Of 309 participants, 21 (7\%) withdrew during the induction period. Among these, five stopped due to lack of efficacy, three for liver events according to pre-specified

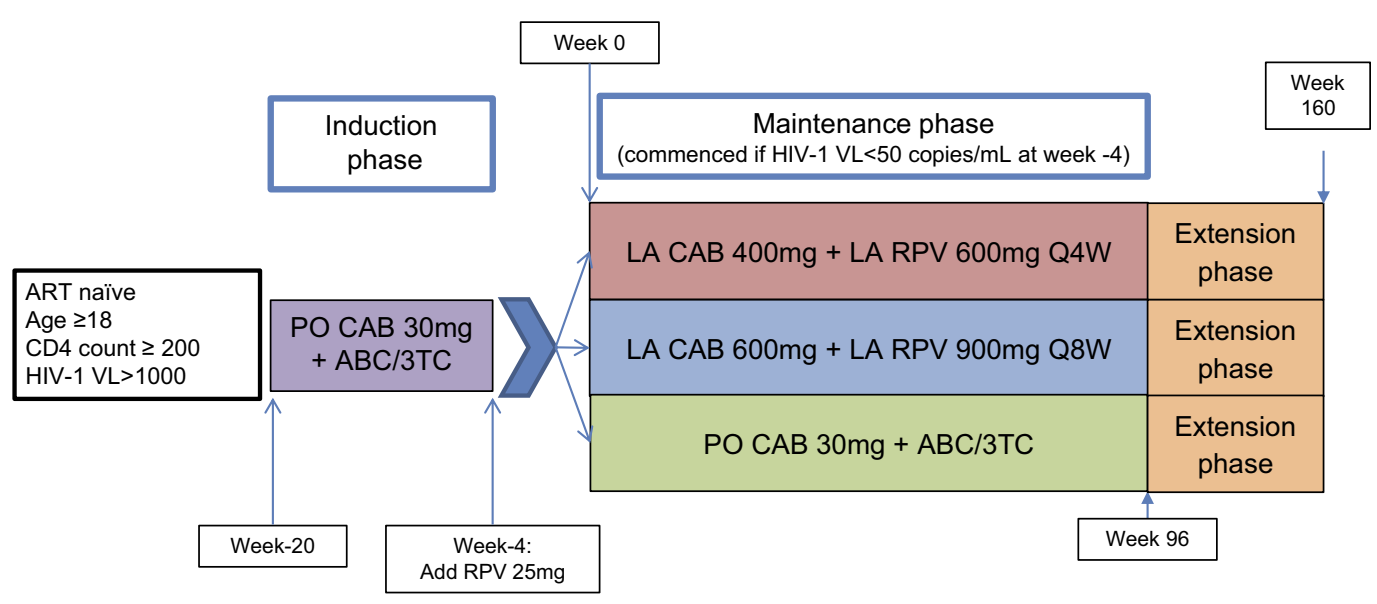

Figure 2 The Long-Acting antireTroviral Treatment Enabling trial - 2 (LATTE-2).

Abbreviations: ART, antiretroviral therapy; HIV, human immunodeficiency virus; ABC, abacavir; 3TC, lamivudine; CAB, cabotegravir; RPV, rilpivirine; Q4W, every 4 weeks; QSW, every 8 weeks; LA, long-acting; PO, oral. 
criteria and three for other AEs. During the maintenance period, 28/286 (10\%) participants had withdrawn by week 96. Ten withdrew due to AEs, of which eight were in the $\mathrm{q} 4 \mathrm{w}$ injectable arm.

There was comparable virological suppression between both injectable arms and the oral treatment arm at week 32 , also seen at weeks 48 and 96 (Table 1). Among three with PDVF, one patient in the $\mathrm{q} 8 \mathrm{w}$ arm had treatment-emergent resistance after 4 weeks with reverse transcriptase (RT) mutations K103N, E138G and K238T conferring lowlevel resistance to rilpivirine, and Q148R mutation conferring high-level resistance to raltegravir, elvitegravir and cabotegravir, but lower-level resistance to dolutegravir and bictegravir. In a second participant in the $\mathrm{q} 8 \mathrm{w}$ arm, the mixture R269R/G was detected at week 48; mutations at codon 269 are not known to decrease cabotegravir susceptibility. $^{27}$

Injection site reactions (ISRs) were the most common AE reported in both injectable arms. Pain occurred in $>95 \%$ of all patients and nodules and swelling in $>25 \%$. Most ISRs were mild and short-lived (median 5 days). ISR frequency decreased from day 1 ( $\geq 85 \%$ in both arms) to approximately half this at week 4 . Headaches were common, in up to $25 \%$ in all arms, although these were mostly mild and not treatment-related.

At week 48, all arms achieved mean trough concentrations greater than the in vitro protein-adjusted $90 \%$ inhibitory concentration for cabotegravir and rilpivirine against HIV-1 wild type $(0.166 \mu \mathrm{g} / \mathrm{mL}$ and $12 \mathrm{ng} / \mathrm{mL}$, respectively). Based on the results of LATTE-2, an optimized $\mathrm{q} 4 \mathrm{w}$ dosing regimen for a future phase III study was selected, although both $\mathrm{q} 4 \mathrm{w}$ and $\mathrm{q} 8 \mathrm{w}$ schedules are undergoing further evaluation. In an extension to 160 weeks, enrolled participants in the injectable arms continued on their current treatment regimen and those on the oral arm who were virologically suppressed were switched to receive an optimized loading dose followed by the $\mathrm{q} 4 \mathrm{w}$ or q8w injectable regimen. At week 160, both IM LA regimens have continued to maintain virological control and no patients have experienced protocol-defined virological failure after week $48 .^{26}$

\section{Phase III studies}

Two phase III trials have now reported 48-week data as conference presentations, both showing LA CR to be noninferior to continued oral triple therapy in terms of the proportion of participants with viremia at that time. ${ }^{28,29}$
FLAIR is an ongoing open-label study enrolling ARTnaïve, hepatitis $B$ surface antigen negative individuals, with no NNRTI RAMs other than K103N (Figure 3). Baseline integrase resistance testing was not done, consistent with current international clinical practice for ART-naïve individuals. On enrolment, all commenced a 20 -week induction phase of the oral fixed-dose combination abacavir, lamivudine and dolutegravir. At week 20, those with HIV RNA $<50$ copies/ $\mathrm{mL}$ as measured at week 16 proceeded to randomization 1:1 to either continue this oral therapy or to switch to a 4-week oral lead-in of CR. After this 4-week period, a loading dose of LA cabotegravir $600 \mathrm{mg}$ and rilpivirine $900 \mathrm{mg}$ was administered, followed by $\mathrm{q} 4 \mathrm{w}$ maintenance doses of IM LA cabotegravir $400 \mathrm{mg}$ and rilpivirine $600 \mathrm{mg}$.

The primary endpoint was proportion with HIV RNA $>50$ copies $/ \mathrm{mL}$ at 48 weeks (ie, 44 weeks after starting injectable therapy), using the required FDA "snapshot" algorithm. Secondary outcomes included resistance at confirmed virological failure, safety and tolerability and patient-reported outcomes. The trial recruited (ITT population) 566 individuals, with 283 in each arm.

For the primary outcome of proportion viremic at 48 weeks (HIV RNA>50 copies/mL), 2.1\% (6/283) in the LA arm and $2.5 \%(7 / 283)$ in oral arm were viremic, showing non-inferiority of LA versus oral triple therapy. There were four $(1.4 \%)$ individuals in the LA treatment arm who discontinued due to lack of efficacy and three $(1.1 \%)$ in the oral treatment arm. There were three participants with confirmed virological failure in the LA treatment arm. Detail available to date on these showed that all three were from Russian study sites and all infected with type A1 virus. At the time of virological failure, one had the NNRTI RAM $\mathrm{E} 138 \mathrm{~K}$, one had the mixture E138E/A/K/T and one K101E. Integrase mutation Q148R was found in two, G140R in one and all showed the integrase polymorphism L74I. However, on testing baseline HIV RNA, the L74I was present in all before treatment start. Alone, L74I does not affect INSTI susceptibility, although it contributes to resistance when found with other mutations, including at position $148 .^{27}$ Drug levels in these three individuals were within the range of those who were virologically suppressed, indicating that injections had been administered and that subtherapeutic levels alone do not explain the failure. Data on the baseline mutations and polymorphisms among those who remained virologically suppressed are needed to compare and we await further information to understand the significance of these descriptive data. 


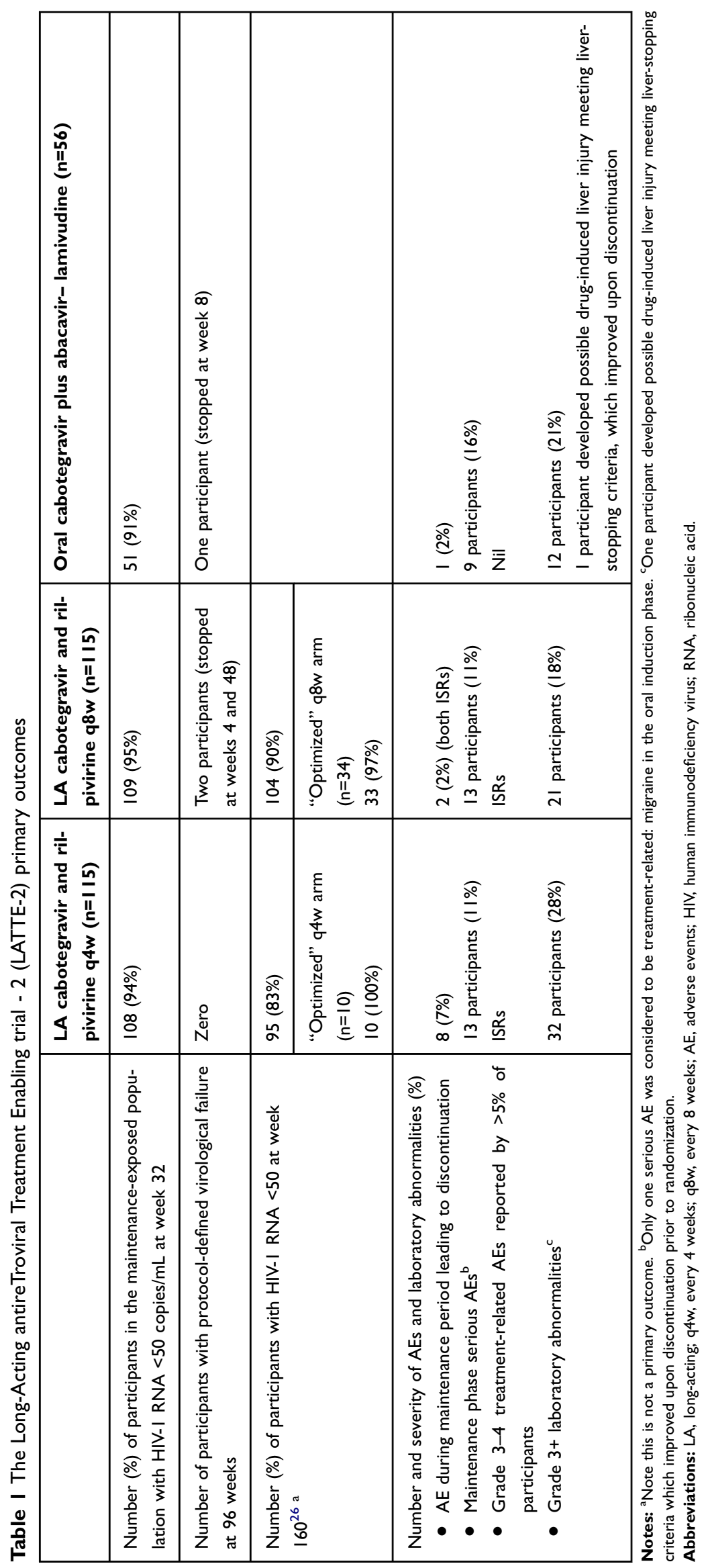



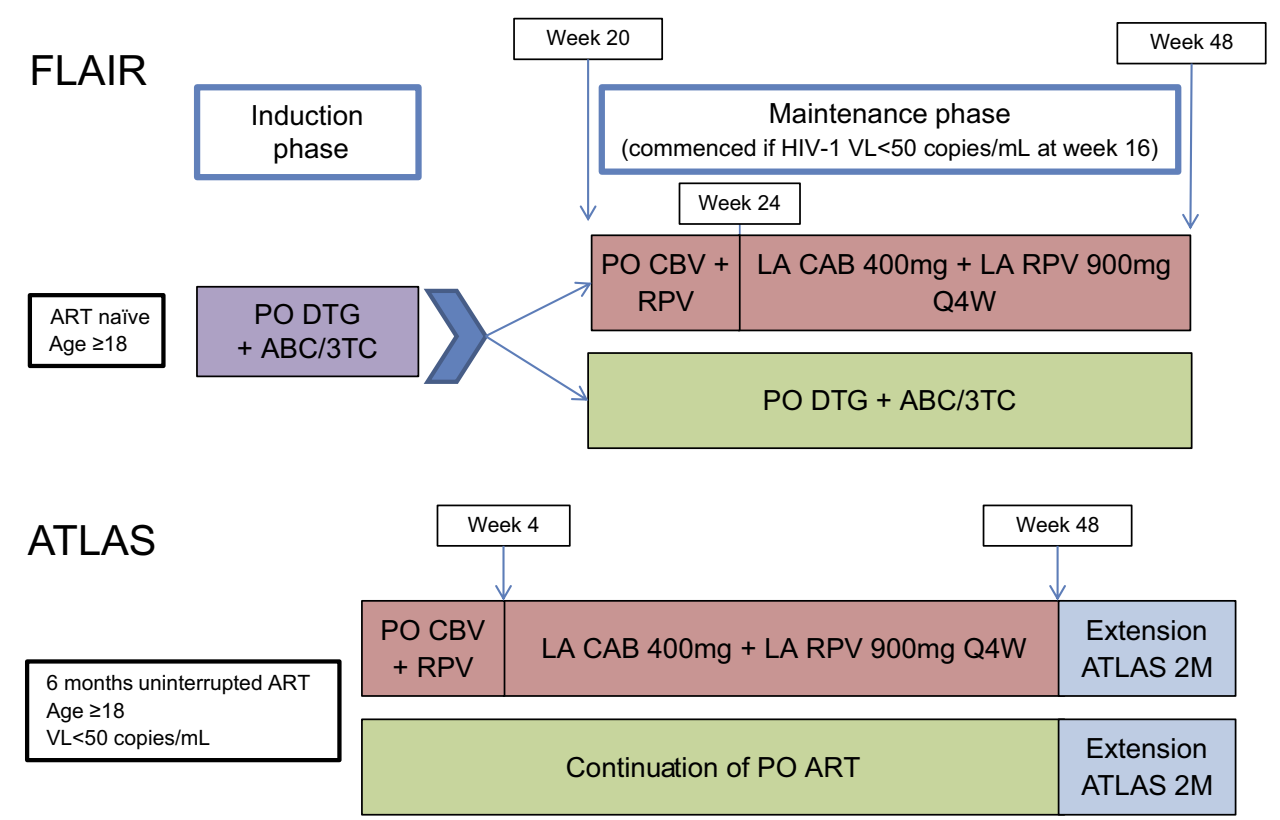

Figure 3 FLAIR and ATLAS.

Abbreviations: ABC, abacavir; 3TC, lamivudine; CAB, cabotegravir; RPV, rilpivirine; OTG, dolutegravir; ART, antiretroviral therapy; Q4W, every 4 weeks; LA, long-acting; PO, oral.

As in LATTE-2, the most common AE was ISR, decreasing in severity and frequency over time. Two participants withdrew from the LA arm because of ISRs, but the majority were non-severe and self-limiting. Participants again reported high levels of satisfaction with the LA treatment. ${ }^{28}$

ATLAS is a phase III, open-label, randomized study recruiting virologically suppressed participants, stable for at least six months on an oral regimen of two NRTIs plus a third agent (protease inhibitor (PI), NNRTI or INSTI). ${ }^{29,30}$ Of note, those on fixed-dose combination abacavir, lamivudine and dolutegravir were excluded and a reason for this is not given. Also excluded were individuals with a prior switch due to treatment failure (HIV-1 RNA $\geq 400$ copies $(\mathrm{mL}$ ), those who have had over one-month break from ART and those with clinically significant comorbidities. This may limit generalizability outside of these groups. Participants with the RT mutation K103N were not excluded. Participants were randomized 1:1 to continue their oral regimen or switch to a 4-week oral lead in phase of daily $\mathrm{CR}$, then proceed to a loading dose and $\mathrm{q} 4 \mathrm{w}$ maintenance LA CR, with the same regimens as used in FLAIR. Data for the 48-week primary endpoint have been presented, but the study is ongoing, including an extension phase of the same $\mathrm{q} 4 \mathrm{w}$ regimen and a study of the $\mathrm{q} 8 \mathrm{w}$ regimen, "ATLAS 2M".

Median prior duration of ART was 4 years, ranging from 1 to 21 years. The third agent was an NNRTI in 50\%.
At week 48, 1.6\% (5/308 participants) in the LA arm and $1.0 \%(3 / 308)$ in the oral arm had virological nonresponse, with HIV RNA $\geq 50$ copies $/ \mathrm{mL}$. The adjusted treatment difference met the definition of non-inferiority. Virological success was seen in $92.5 \%$ (285/308) participants in the LA therapy arm, compared with $95.5 \%(294 / 308)$ in the oral therapy arm.

As seen in FLAIR, there was some resistance emerging at failure in the LA therapy arm and further study on this is warranted. To date, we know that two were from Russian and one from a French study site; all had subtype A viruses (two A/A1 in Russia, one AG in France). RNA-based resistance testing at failure showed the RT mutation E138A, E or K, associated with rilpivirine resistance, in all three, with V108I in one. On integrase testing, L74I was seen in two, of whom one also had $\mathrm{N} 155 \mathrm{H}$, associated with a 2.7 -fold change in susceptibility to cabotegravir. As in FLAIR, drug levels were below mean for the study population, but within range for most participants who maintained virological suppression. Comparing with baseline DNA (deoxyribonucleic acid) resistance testing (undertaken on stored samples, since all participants had HIV RNA $<50$ at baseline), E138 mutations were seen in two participants in baseline samples and L74I in two. Data on baseline DNA mutations among those with virological suppression at 48 weeks are not yet available and we should interpret DNA resistance testing with caution since it is not widely used in clinical practice and may include 
non-replicative viral $\mathrm{DNA}^{31}$ although resistance in viral DNA has been associated with future virological failure in some individuals. ${ }^{32}$

AEs were similar to those seen in FLAIR, with more drug-related AEs in the LA treatment arm (as would be expected in a study in which participants stable for six months on an oral regimen are randomized to switch) and headache reported by $11 \%$ in the LA treatment arm, versus $6 \%$ in the oral therapy arm. Only 10 participants in the LA treatment arm and 5 in the oral therapy arm withdrew due to AEs. Again, ISRs occurred and decreased in frequency as the study progressed and $99 \%$ were grade $1-2$. There were few discontinuations due to ISRs and again those switching to injectable therapy expressed high levels of satisfaction with the treatment.

\section{Participant satisfaction and qualitative data: "it might be painful, but it is better than pills,33}

The HIV Treatment Satisfaction Questionnaire (HIVTSQ) is a 10 -item validated questionnaire developed to evaluate HIV therapy. It addresses issues such as perceived convenience, flexibility and understanding of treatment as well as whether PLWH would be satisfied to continue their current form of treatment or would recommend it to others. The original status version (HIVTSQs) has since been updated to the new change version (HIVTSQc) that better allows tracking of change in satisfaction, but both are validated for use in clinical trials. ${ }^{34,35}$ A 12-item HIVTSQ (HIVTSQ-12) has now been created to address the discomfort caused by therapy and its ease of use. ${ }^{36}$

In LATTE-2, the HIVTSQ was used to assess participant satisfaction with injectable versus ongoing oral treatment. High levels of satisfaction were recorded, with $98 \%$ and $99 \%$ of the participants in injectable arms reporting that they were satisfied or very satisfied to continue at week 48 , compared with $88 \%$ in the oral arm. This is despite ISRs, although of course in a preselected population who agreed to randomization to injectable therapy. ${ }^{25}$

A separately published qualitative study of LATTE-2 participants in the US and Spain provided in-depth interview data from 27 participants (including only two females) and 12 providers. ${ }^{33}$ Key conclusions were that participants reported convenience of LA injections and benefited from the emotional advantage of reduced risk of inadvertent disclosure and eliminating the daily reminder of living with HIV. Benefits in terms of stigma and discrimination were cited, with reduced concern about travel to countries where laws still discriminate on the basis of HIV status.

However, participants did express concern regarding the increased frequency of clinic visits, with colleagues noticing time off, and many hoped for less frequent injections to be available. Participant views were largely positive, albeit among those choosing to participate in the trial, experiencing virological suppression on LA therapy and then agreeing to participate in a qualitative sub-study.

Providers of injectable therapy were not so selected and had some concerns about the characteristics of PLWH who would most benefit from injectables and the fact that injectable therapy does not completely obviate the need for adherence to a medication schedule. Other concerns included emergent resistance if injections are missed and issues with switching if necessitated by new concomitant medications.

ATLAS and FLAIR have also used the HIVTSQ to determine participant satisfaction with injectable therapy with participants in both studies demonstrating markedly higher levels of satisfaction with LA regimens compared to oral therapy. In those participants who switched from oral to injectable therapy in both studies, the vast majority of responding participants (99\% in FLAIR and $97 \%$ in ATLAS) preferred injectable therapy. ${ }^{28,29}$

\section{Drug tolerability}

LA injectable therapy is presumed advantageous to oral therapy in part because of better tolerability, higher patient satisfaction and reduced need for daily adherence. Rilpivirine has a favorable safety profile compared to older NRTIs, with lower rates of AEs and discontinuations compared to efavirenz in its licensing studies. ${ }^{12,13}$ The most frequent $\mathrm{AE}$ reported with injectable cabotegravir for both treatment and pre-exposure prophylaxis is mild ISR. ${ }^{25,37,38}$ Despite LATTE detecting a higher frequency of headaches and depression in participants on oral cabotegravir versus efavirenz, only headaches were reported to be treatmentrelated and at a frequency of $>10 \%{ }^{24}$ with only $5-6 \%$ of the patients in LATTE-2 suffering treatment-related headaches. ${ }^{25}$ Other potential neurocognitive side-effects (depression, memory impairment, asthenia and myalgia, and anxiety) were observed in the cabotegravir group in ATLAS causing treatment discontinuation but were not felt to be drug-related. ${ }^{29}$ The neurocognitive AEs seen with dolutegravir post-licensing have not, to date, been reported with cabotegravir. ${ }^{39-43}$ 
Weight gain has now been reported as a potential complication of INSTI use, ${ }^{44}$ To date, pre-exposure prophylaxis (PrEP) studies have found no increase in weight gain with cabotegravir compared to placebo, ${ }^{45}$ but further data on this are needed from treatment studies.

\section{Drug resistance in in vitro and in vivo studies}

Two key aspects to consider are: first, the suitability of CR LA therapy for individuals with pre-existing RAMs or with experience of several ART regimens before moving to longacting injectables; second, the frequency and nature of resistance emerging on treatment and a consideration of what treatment combinations may be effective after failure.

In the licensing studies for rilpivirine, ECHO and THRIVE, ${ }^{12,13}$ and similarly in the STAR study, ${ }^{46}$ treatment-naïve adults with any of 39 NNRTI mutations at baseline were excluded, including $\mathrm{K} 103 \mathrm{H} / \mathrm{N} / \mathrm{S} / \mathrm{T}$, which was appropriate since the comparator arm was an efavirenz-based regimen. Similarly, in the studies of oral dolutegravir and rilpivirine, SWORD-1 and SWORD-2, ${ }^{47}$ in which individuals who were stable on ART were randomized to switch to the dual therapy or continue triple therapy, exclusion criteria included any previous switch because of virological failure and evidence of major mutations associated with resistance to PI, INSTI, NRTI or NNRTI groups. This limits the generalizability of the data to more treatment-experienced individuals, particularly those with long treatment histories including the first-generation NNRTIs or dual NRTI therapy. The WISARD study, now recruiting, will investigate the efficacy of dolutegravir and rilpivirine dual therapy among individuals with the $\mathrm{K} 103 \mathrm{~N}$ mutation and should provide useful data for that oral regimen. ${ }^{48}$ However, we must be cautious in extrapolating results of dolutegravir-based studies to cabotegravir, since they are not identical, particularly in terms of resistance profile. Both LATTE studies also excluded individuals with major RAMs.

In terms of treatment-emergent resistance, we await further data on those experiencing virological failure in ATLAS and FLAIR. We note that in the rilpivirine licensing studies the most frequent emerging rilpivirine-associated RAM was E138K. ${ }^{49}$ Considering potential treatment after virological failure on $\mathrm{CR}$, individualized regimens would have to be constructed depending on RAMs found and susceptibility to agents with high genetic barrier to resistance.
In vitro studies may indicate potential for the development of resistance to cabotegravir following virological failure on first-generation INSTIs, with novel mutations C56S and G149A occurring on passage of Q148H virus (indicating resistance to raltegravir and elvitegravir) in the presence of cabotegravir. ${ }^{50}$ In vitro activity of cabotegravir against HIV-1 isolates with mutations affecting raltegravir and elvitegravir appears lower than that of dolutegravir and bictegravir, particularly against raltegravir-resistant isolates with G140S or Q148H. ${ }^{51}$ Cabotegravir is consistently shown to be less potent than bictegravir and dolutegravir in vitro, with the Q148R/K seen in clinical trials resulting in cross-resistance across the INSTI class. ${ }^{52}$

\section{Therapeutic potential in differing patient populations ART-experienced patients or with advanced HIV infection (CD4 $\leq 200$ )}

Both LATTE and LATTE-2 excluded treatment-experienced individuals, those with major RAMs and those with active complications from late-stage HIV. ${ }^{24,25}$ Enrolled participants had relatively high median CD4 counts in both studies, as individuals with baseline CD4 counts $<200$ cells $/ \mu \mathrm{L}$ were excluded. In LATTE, across CR arms there were lower proportions with baseline HIV-1 RNA $\geq 100,000$ copies/mL versus $<100,000$ that achieved virological suppression at week 96 , but numbers in each group were small. ${ }^{24}$ Therefore, the use of injectable CR in ART-experienced individuals or those with low CD4 counts is yet to be evidenced.

LATTE-2 included a sub-study that analyzed paired plasma and cerebrospinal fluid (CSF) taken seven days after an LA CR dose (15 participants receiving cabotegravir q8w and three receiving cabotegravir q4w). CSF cabotegravir concentrations positively correlated with total plasma cabotegravir concentrations. Both cabotegravir and rilpivirine concentrations exceeded their in vitro $50 \%$ inhibitory concentration $\left(\mathrm{IC}_{50}\right)$ for wild-type HIV-1 in all but one patient with a CSF rilpivirine concentration of $<1 \mathrm{ng} / \mathrm{mL}$ (rilpivirine $\mathrm{IC}_{50} 0.081 \mathrm{ng} / \mathrm{mL}$ ). All patients maintained high rates of virological suppression in plasma and CSF with only one patient in the $\mathrm{q} 8 \mathrm{w}$ arm having detectable CSF HIV RNA on the SuperLow assay (detects HIV-1 RNA $>2$ cells $/ \mathrm{mL}$ ) but undetectable on the Abbott real-time assay (HIV-1 RNA $<50$ cells $/ \mathrm{mL}){ }^{53}$ Thus, cabotegravir and rilpivirine seem to effectively penetrate the CSF and are able to successfully suppress HIV replication in this sanctuary site making it a 
suitable regimen for those with advanced infection and possible or definite CNS opportunistic infections (OIs).

\section{Individuals with no enteral route}

At present only the fusion inhibitor enfuvirtide (T20) and the NRTI zidovudine are available in injectable form and neither are feasible now for maintenance therapy. LA injectables may provide a much-needed option for the few individuals with poor gastrointestinal absorption due to severe illness, swallowing problems or comorbidities although its data on its use in this context will probably only be available post-marketing.

\section{Individuals with suboptimal adherence to oral therapy}

The potential benefit to this group is yet to be demonstrated in studies, but the potential to maintain virological suppression where oral therapy has not would of course be beneficial. In some with chaotic lifestyles or addictions, LA injectables may not be the answer. Concerns include missing or delayed injections, causing reduction in blood levels of drug to below those required for virological suppression and leading to resistance that threatens future options. Strategies such as oral CR or other drugs, for example, PIs, to cover this pharmacokinetic "tail" are not yet evidenced but are needed. For those individuals for whom daily oral therapy presents a psychological burden, but who maintain engagement with clinical care, LA injectables could present a solution. However, studies to date have all used an oral lead-in period and starting injectable therapy without this, or in a true ART-naïve population, is not yet evidenced. There is still a knowledge gap in the efficacy of LA therapy in patients with prior treatment-failure or those with treatment breaks as they have been excluded from trials. ${ }^{30}$

\section{Individuals requiring NRTI-sparing regimens}

Despite the appeal of two-drug regimens, not all combinations are equally efficacious or non-inferior to triple-drug combinations, and some may cause use-limiting toxicity. ${ }^{54}$ $\mathrm{CR}$ has the advantage of avoiding long-term toxicity of NRTIs and the toxicity and drug interactions of PIs. ${ }^{55,56}$ LA CR is a tenofovir disoproxil (TDF)-sparing regimen that reduces pill burden and eliminates the renal and bone risks associated with long-term TDF use. However, the role of tenofovir-sparing regimens with the availability now of tenofovir alafenamide (TAF) is less clear.

\section{Individuals with co-infections and comorbidities}

Most participants in LATTE and LATTE-2 had no significant comorbidities. Both studies included a small number of hepatitis C RNA positive individuals, although hepatitis $\mathrm{B}$ infection was an exclusion criterion in both because of the tenofovir-free regimens. ${ }^{24,25}$ Treatment of hepatitis C in individuals on LA CR regimens may involve interactions between cabotegravir and antivirals for hepatitis $\mathrm{C}$, which need to be studied.

Cabotegravir is highly bound to albumin with less than $1 \%$ free drug in circulation ${ }^{57}$ and is primarily eliminated via the predominantly hepatic UGT (uridine 5 '-diphosphoglucurnosyltransferase) metabolism pathway (mainly UGT1A1, with a contribution from UGT1A9). ${ }^{58}$ No dose-adjustment appears to be required in mild to moderate hepatic impairment. ${ }^{57}$ Two individuals receiving oral cabotegravir in LATTE and LATTE-2 met stopping criteria for liver disease considered possibly drug-related. ${ }^{24,25}$ Therefore, concern still stands regarding cabotegravir in known severe pre-existing liver disease, viral hepatitis, or those on concomitant medications.

Despite patients with severe renal impairment being excluded from treatment studies, a phase I study showed oral cabotegravir may be administered without dose adjustment in severe renal impairment (creatinine clearance $<30 \mathrm{~mL} / \mathrm{min}$, not on renal replacement therapy). ${ }^{59}$ Cabotegravir is also considered to not affect cardiac repolarisation although studies have only been done in healthy subjects receiving oral cabotegravir. ${ }^{60}$ Data are lacking on the use of rilpivirine in severe renal or hepatic impairment and manufacturers recommend it is used with caution. ${ }^{22}$

\section{Drug-drug interactions (DDIs)}

Known DDIs with oral rilpivirine include proton-pump inhibitors (presumably not a concern in injectable therapy), dexamethasone, rifampicin, phenytoin and carbamazepine. ${ }^{22}$ In vitro studies found cabotegravir interacts with organic ion transporter (OAT) 1 or OAT3 substrates with a small therapeutic window such as methotrexate; ${ }^{61}$ however, physiologically based pharmacokinetic (PBPK) models suggest no dose adjustment is required. ${ }^{62}$ Rifampicin has been demonstrated to induce oral cabotegravir metabolism, significantly decreasing cabotegravir exposure, and this result is expected in 
injectable LA preparations as well. ${ }^{63,64}$ Rifampicin is a cornerstone in anti-tuberculous (TB) therapy and therefore CR would currently be contraindicated in those requiring TB treatment. This is a key issue where TB incidence is high and where coinfection is frequent.

\section{Data in females}

Trial participants are predominantly male: LATTE and LATTE 2 participants were $96 \%$ and $92 \%$ male, respectively, and both studies excluded pregnant women. ${ }^{24}$ Phase III studies have recruited more women: $22 \%$ in FLAIR and $33 \%$ in ATLAS, although numbers are unlikely to be sufficient to determine any differences in response by sex. $^{28,29}$

Of 200 participants in the PrEP cabotegravir study HPTN $077,{ }^{37} 66 \%$ were female. HPTN 077 showed no difference in proportions completing the regimen, similar numbers of AEs leading to discontinuation, and similar frequency of ISRs between females and males. There were some pharmacokinetic differences, with females, unlike males, consistently achieving target trough concentrations, and in tail-phase kinetics where it is anticipated that the terminal decay rate is $50 \%$ slower in females. A healthy volunteer study of LA rilpivirine has demonstrated that females have lower peak rilpivirine concentrations in both serum and genital fluids. ${ }^{18}$ More data on the pharmacokinetics of injectable CR in females are needed from treatment trials to address the knowledge gaps in dosing, safety, efficacy and tolerability.

There is no interaction between cabotegravir and levonorgestrel or ethinyl oestradiol-containing oral contraceptives in healthy adult women ${ }^{65}$ nor was a change in cabotegravir concentrations detected with any form of hormonal contraception in women in the HPTN 077 study. ${ }^{66}$ To date, we are not aware of any data on interactions between LA CR and depot contraceptives.

There are no studies of LA CR in pregnant women. Increased activity of UGT1A1 and UGT1A9 during pregnancy may lead to increased cabotegravir clearance, although the effect on drug concentration is unclear. ${ }^{67}$ Considering other INSTIs, there are proven changes in raltegravir pharmacokinetics in pregnancy prompting an increase in the recommended dose compared to non-pregnant females, ${ }^{68}$ however, no dose adjustment is required for dolutegravir. Raltegravir has been favored in international pregnancy guidelines in women with high baseline $\mathrm{VL}$, requiring treatment intensification, or presenting in late pregnancy. ${ }^{69,70}$ There is some evidence that INSTIs contribute to greater likelihood of achieving undetectable HIV RNA at delivery. ${ }^{71,72}$

Concern regarding teratogenicity of dolutegravir has prompted recommendations to avoid it for women considering conception and during the first trimester. ${ }^{69,70}$ Given the lack of safety data in pregnant women and the long $t_{1 / 2}$ of LA therapy, it cannot be recommended in those situations at present.

\section{Children and adolescents}

There are restrictions on the use of INSTIs and rilpivirine in the pediatric population due to lack of data. There is one in silico PBPK dose prediction study that has provided dosing recommendations of LA CR in children and adolescents weighing $15-70 \mathrm{~kg}^{73}$ No dose prediction studies have been undertaken in children weighing less than $15 \mathrm{~kg}$. A pharmacokinetic and safety study of LA CR in virologically suppressed adolescents aged 12-18, MOCHA, is currently in progress. ${ }^{74}$ Of importance will be the acceptability of frequent injections in this group. This is particularly pertinent in younger children, in whom future studies may be required.

\section{Role in low- and middle-income countries (LMIC)}

The vast majority of study sites in HIV treatment studies of LA therapy are based in high-income countries (HIC) in predominantly white populations. This leaves large knowledge gaps of its efficacy and safety in those of other ethnic backgrounds. Drug interactions discussed earlier are among the challenges in the rollout of LA CR in countries with a high incidence of TB.

Determinants of drug acceptability and adherence vary across the globe and in different patient populations. ${ }^{75-77}$ Factors such as beliefs about ART, stigma, social support or depressive symptoms are important determinants of adherence $^{78}$ and strategies to address them should be relevant to the local context. Where HIV is still highly stigmatized, injectable therapy may allow PLWH more easily to conceal treatments from others, reducing risk of inadvertent disclosure.

To date, health care professionals have delivered LA ART $^{33}$ This will be a challenge in clinics with a large number of PLWH where injectables will require more clinical time and in services covering wide geographical areas. Strategies involving decentralized services or nonhealth care workers administering injections may help. 
Requirement of cold chain and storage at $2-8^{\circ} \mathrm{C}$ may also affect LA CR distribution in these settings. ${ }^{79}$

\section{Outstanding questions and future work on cabotegravir/rilpivirine}

While we now have 48-week data from the first phase III clinical trials, there are ongoing trials yet to report and follow-up data on the FLAIR and ATLAS studies will give valuable information on durability of the regimen and resistance emerging on treatment. Key information will come from the ATLAS-2M study, evaluating the efficacy of the $\mathrm{q} 8 \mathrm{w}$ regimen, which may provide practical, economic and tolerability benefit over $\mathrm{q} 4 \mathrm{w} .{ }^{80}$ In addition, the MOCHA study in adolescents has potential to provide data for much-needed easier adherence ${ }^{74}$ and the phase III study NCT03635788, enrolling participants with a history of difficulty adhering to oral treatment regimens should help define the role of IM LA therapy in this population. ${ }^{81}$ Importantly, there are outstanding knowledge gaps in the use of injectable CR for HIV treatment in females and its use in pregnancy cannot be recommended at present.

Real-world data will be essential in defining the groups of individuals most likely to benefit from LA therapy; with those with previous NNRTI resistance, those with frequent non-attendance at the clinic and those with previous adherence difficulties are key populations who may stand to benefit. Further data on the "tail" after a missed injection and advice on how to manage this are needed as well as is data on the safety of therapy without the oral lead-in. Pharmacokinetics and drug interactions will be key among those with OIs, or who develop OIs while on therapy, since switching from LA regimens presents challenges. We await further data on whether the neuropsychiatric SE of dolutegravir and the weight gain potentially attributable to INSTIs are seen with cabotegravir.

In conclusion, we proceed with caution into this new era of HIV therapy, which could lift the burden of daily oral therapy for many people living with HIV, but which must be supported by robust safety and durability data as well as real-world cohorts.

\section{Disclosure}

CLvH has received conference scholarship from Viiv and has taken part in an advisory board for Viiv. She has received travel grants from Janssen and speaker fees from Gilead. CF reports no conflicts of interest in this work.

\section{References}

1. Joint United Nations Programme on HIV \& AIDS. UNAIDS Data 2018. Geneva, Switzerland: Joint United Nations Programme on HIV/AIDS (UNAIDS); 2018. Available from: https://www.aidsdata hub.org/sites/default/files/publication/UNAIDS_Data_2018.pdf

2. Joint United Nations Programme on HIV/AIDS (UNÄIDS). 90-90-90 an Ambitious Treatment Target to Help End the AIDS Epidemic. Geneva, Switzerland: Joint United Nations Programme on HIV/ AIDS (UNAIDS); Vol. 1.; 2014.

3. Harris TG, Rabkin M, El-Sadr WM. Achieving the fourth 90: healthy aging for people living with HIV. AIDS. 2018;32(12):1563-1569. doi:10.1097/QAD.0000000000001870

4. Lazarus JV, Safreed-Harmon K, Barton SE, et al. Beyond viral suppression of HIV - the new quality of life frontier. BMC Med. 2016;14(1):94. doi:10.1186/s12916-016-0640-4

5. Whitfield T, Torkington A, van Halsema C. Profile of cabotegravir and its potential in the treatment and prevention of HIV-1 infection: evidence to date. HIV/AIDS - Res Palliat Care. 2016;8:157-164. doi:10.2147/HIV.S97920

6. Ferretti F, Boffito M. Rilpivirine long-acting for the prevention and treatment of HIV infection. Curr Opin HIV AIDS. 2018;13(4):300307. doi: $10.1097 / \mathrm{COH} .0000000000000474$

7. ViiV Healthcare. GSK1265744 (Cabotegravir, CAB) for named patient/compassionate use in HIV. ClinicalTrials.gov. Available from: https://clinicaltrials.gov/ct2/show/NCT03462810. Published 2018. Accessed April 30, 2019.

8. Spreen W, Min S, Ford SL, et al. Pharmacokinetics, safety, and monotherapy antiviral activity of GSK1265744, an HIV integrase strand transfer inhibitor. HIV Clin Trials. 2013;14(5):192-203. doi:10.1310/hct1405-192

9. Spreen W, Ford SL, Chen S, et al. GSK1265744 pharmacokinetics in plasma and tissue after single-dose long-acting injectable administration in healthy subjects. J Acquir Immune Defic Syndr. 2014;67 (5):481-486. doi:10.1097/QAI.0000000000000301

10. Committee for Medicinal Products for Human Use. Edurant Rilpivirine. Amsterdam, the Netherlands: European Medicines Agency; 2011. Available from: https://www.ema.europa.eu/en/medi cines/human/EPAR/edurant. Accessed January 22, 2019.

11. Food and Drug Administration. Rilpivirine. Maryland, USA: AIDSinfo; 2011. Available from: https://aidsinfo.nih.gov/drugs/426/ rilpivirine/19/professional. Accessed April 30, 2019.

12. Molina J-M, Cahn P, Grinsztejn B, et al. Rilpivirine versus efavirenz with tenofovir and emtricitabine in treatment-naive adults infected with HIV-1 (ECHO): a phase 3 randomised double-blind activecontrolled trial. Lancet (London, England). 2011;378(9787):238246. doi:10.1016/S0140-6736(11)60936-7

13. Cohen CJ, Andrade-Villanueva J, Clotet B, et al. Rilpivirine versus efavirenz with two background nucleoside or nucleotide reverse transcriptase inhibitors in treatment-naive adults infected with HIV1 (THRIVE): a phase 3, randomised, non-inferiority trial. Lancet. 2011;378(9787):229-237. doi:10.1016/S0140-6736(11)60983-5

14. Healthcare V. Juluca $50 \mathrm{mg} / 25 \mathrm{mg}$ film-coated tablets. electronic Medicines Compendium; 2019. Available from: https://www.medi cines.org.uk/emc/product/9246/smpc. Accessed May 2, 2019.

15. Gilead Sciences. Odefsey $200 \mathrm{mg} / 25 \mathrm{mg} / 25 \mathrm{mg}$ film-coated tablets. electronic Medicines Compendium; 2018. Available from: https://www.medi cines.org.uk/emc/product/7262/smpc. Accessed May 2, 2019.

16. Gilead Sciences. Eviplera $200 \mathrm{Mg} / 25 \mathrm{Mg} / 245 \mathrm{Mg}$ Film Coated Tablets. Surrey, UK: Electronic Medicines Compendium; 2018. Available from: https://www.medicines.org.uk/emc/product/2764/ smpc. Accessed May 2, 2019.

17. Janssen Pharmaceuticals. Pre-Approval Access to Rilpivirine Long Acting (RPV LA) Injectable Suspension for the Treatment of a Participant with Human Immunodeficiency Virus (HIV)-1 Infection. Maryland, USA: AIDSinfo; 2019. Available from: https://aidsinfo.nih.gov/clinicaltrials/details/NCT03847376. Accessed April 30, 2019. 
18. Jackson AGA, Else LJ, Mesquita PMM, et al. A compartmental pharmacokinetic evaluation of long-acting rilpivirine in HIV-negative volunteers for pre-exposure prophylaxis. Clin Pharmacol Ther. 2014;96(3):314-323. doi:10.1038/clpt.2014.118

19. Verloes R, Deleu S, Niemeijer N, Crauwels H, Meyvisch P, Williams P. Safety, tolerability and pharmacokinetics of rilpivirine following administration of a long-acting formulation in healthy volunteers. HIV Med. 2015;16(8):477-484. doi:10.1111/hiv.12247

20. McGowan I, Dezzutti CS, Siegel A, et al. Long-acting rilpivirine as potential pre-exposure prophylaxis for HIV-1 prevention (the MWRI01 study): an open-label, phase 1, compartmental, pharmacokinetic and pharmacodynamic assessment. Lancet HIV. 2016;3(12):e569e578. doi:10.1016/S2352-3018(16)30113-8

21. University of Liverpool. HIV drug interactions. Available from: https://www.hiv-druginteractions.org/checker. Published 2019. Accessed April 30, 2019.

22. Janssen Pharmaceuticals. Edurant 25 Mg Tablets - Summary of Product Characteristics. Surrey, UK: Electronic Medicines Compendium; 2018. Available from: https://www.medicines.org.uk/emc/product/4968/ smpc\#companyDetails. AccessedJanuary 22, 2019.

23. Ford SL, Gould E, Chen S, et al. Lack of pharmacokinetic interaction between rilpivirine and integrase inhibitors dolutegravir and GSK1265744. Antimicrob Agents Chemother. 2013;57(11):54725477. doi:10.1128/AAC.01235-13

24. Margolis DA, Brinson CC, Smith GHR, et al. Cabotegravir plus rilpivirine, once a day, after induction with cabotegravir plus nucleoside reverse transcriptase inhibitors in antiretroviral-naive adults with HIV-1 infection (LATTE): a randomised, phase 2b, dose-ranging trial. Lancet Infect Dis. 2015;15(10):1145-1155. doi:10.1016/ S1473-3099(15)00152-8

25. Margolis DA, Gonzalez-Garcia J, Stellbrink HJ, et al. Long-acting intramuscular cabotegravir and rilpivirine in adults with HIV-1 infection (LATTE-2): 96-week results of a randomised, open-label, phase 2b, non-inferiority trial. Lancet. 2017;390(10101):1499-1510. doi:10.1016/S0140-6736(17)31917-7

26. Margolis D, Gonzalez-Garcia J, Stellbrink H-J, et al. Safety, efficacy and durability of long-acting cabotegravir and rilpivirine as two-drug IM maintenance therapy for HIV-1 infection: LATTE-2 week 160 results. In: HIV Glasgow. Glasgow; 2018:Poster 118.

27. Stanford University. HIV drug resistance database. 2019. Available from: https://hivdb.stanford.edu/. Accessed April 30, 2019.

28. Orkin C, Arastéh K, Hernández-Mora Gorgolas M, et al. Long acting cabortegravir + rilpivirine for HIV maintenance: FLAIR week 48 results. Conference on Retroviruses and Opportunistic Infections; 2019:Abstract 140. Seattle.

29. Swindells S, J-F A-V, Richmond GJ, et al. Long-acting cabotegravir + rilpivirine as maintenance therapy: atlas week 48 resultS. Conference on Retroviruses and Opportunistic Infections; 2019:Abstract 139. Seattle.

30. ViiV Healthcare. Study evaluating the efficacy, safety, and tolerability of switching to long-acting cabotegravir plus long-acting rilpivirine from current antiretroviral regimen in virologically suppressed HIV-1-infected adults. ClinicalTrials.gov. Available from: https://clinicaltrials.gov/ct2/ show/NCT02951052. Published 2018. Accessed January 31, 2019.

31. Allavena C, Rodallec A, Leplat A, et al. Interest of proviral HIV-1 DNA genotypic resistance testing in virologically suppressed patients candidate for maintenance therapy. J Virol Methods. 2018;251:106110. doi:10.1016/j.jviromet.2017.10.016

32. Armenia D, Zaccarelli M, Borghi V, et al. Resistance detected in PBMCs predicts virological rebound in HIV-1 suppressed patients switching treatment. J Clin Virol. 2018;104:61-64. doi:10.1016/j. jcv.2018.04.001

33. Kerrigan D, Mantsios A, Gorgolas M, et al. Experiences with long acting injectable ART: A qualitative study among PLHIV participating in a Phase II study of cabotegravir + rilpivirine (LATTE-2) in the United States and Spain. PLoS One. 2018;13(1):e0190487. doi:10.1371/journal.pone. 0190487
34. Woodcock A, Psychol C, Bradley C. Validation of the revised 10item HIV treatment satisfaction questionnaire status version and new change version. Value Heal. 2006;9(5):320-333. doi:10.1111/j.15244733.2006.00121.x

35. Woodcock A, Bradley C. Validation of the HIV treatment satisfaction questionnaire (HIVTSQ). Qual Life Res. 2001;10(6):517-531. AccessedApril 22, 2019. doi:10.1023/A:1013050904635

36. Romaine J, Murray M, Bradley C. Psychometric evaluation of the revised hiv treatment satisfaction questionnaire (Hivtsq). Value Heal. 2016;19(7):A420. doi:10.1016/j.jval.2016.09.426

37. Landovitz RJ, Li S, Grinsztejn B, et al. Safety, tolerability, and pharmacokinetics of long-acting injectable cabotegravir in low-risk HIV-uninfected individuals: HPTN 077, a phase 2a randomized controlled trial. PLoS Med. 2018; 15(11):e1002690. Newell M-L, ed. doi:10.1371/journal.pmed.1002593

38. Murray MI, Markowitz M, Frank I, et al. Satisfaction and acceptability of cabotegravir long-acting injectable suspension for prevention of HIV: patient perspectives from the ECLAIR trial. HIV Clin Trials. 2018;19(4):129-138. doi:10.1080/15284336.2018.1511346

39. Cuzin L, Pugliese P, Katlama C, et al. Integrase strand transfer inhibitors and neuropsychiatric adverse events in a large prospective cohort. J Antimicrob Chemother. 2019;74(3):754-760. doi:10.1093/ jac/dky497

40. MGJ DB, GEL VDB, van Holten N, et al. Intolerance of dolutegravircontaining combination antiretroviral therapy regimens in real-life clinical practice. AIDS. 2016;30(18):2831-2834. doi:10.1097/ QAD.0000000000001279

41. Fettiplace A, Stainsby C, Winston A, et al. Psychiatric symptoms in patients receiving dolutegravir. JAIDS J Acquir Immune Defic Syndr. 2017;74(4):423-431. doi:10.1097/QAI.0000000000001269

42. Hoffmann C, Welz T, Sabranski M, et al. Higher rates of neuropsychiatric adverse events leading to dolutegravir discontinuation in women and older patients. HIV Med. 2017;18(1):56-63. doi:10.1111/ hiv. 12468

43. Peñafiel J, de Lazzari E, Padilla M, et al. Tolerability of integrase inhibitors in a real-life setting. $J$ Antimicrob Chemother. 2017;72 (6):1752-1759. doi:10.1093/jac/dkx053

44. Bourgi K, Jenkins C, Rebeiro PF, et al. Greater weight gain among treatment-naive persons starting integrase inhibitors. Conference on Retroviruses and Opportunistic Infections; 2019:Abstract 670. Seattle.

45. Landovitz RJ, Zangeneh SZ, Chau G, et al. Cabotegravir is not associated with weight gain in HIV-negative individuals: HPTN 077. Conference on Retroviruses and Opportunistic Infections; 2019:Abstract 34. Seattle.

46. Cohen C, Wohl D, Arribas JR, et al. Week 48 results from a randomized clinical trial of rilpivirine/emtricitabine/tenofovir disoproxil fumarate vs. efavirenz/emtricitabine/tenofovir disoproxil fumarate in treatment-naive HIV-1-infected adults. AIDS. 2014;28(7):989-997. doi:10.1097/QAD.0000000000000169

47. Llibre JM, Hung -C-C, Brinson C, et al. Efficacy, safety, and tolerability of dolutegravir-rilpivirine for the maintenance of virological suppression in adults with HIV-1: phase 3, randomised, non-inferiority SWORD-1 and SWORD-2 studies. Lancet. 2018;391 (10123):839-849. doi:10.1016/S0140-6736(17)33095-7

48. European AIDS Treatment Network Infectious Disease Foundation. WISARD: Switch study mutation rilpivirine dolutegravir. Eu Clinical Trials Register. Available from: https://www.clinicaltrialsregister.eu/ ctr-search/trial/2017-004040-38/ES. Published 2018. Accessed April 30, 2019.

49. Cohen CJ, Molina J-M, Cassetti I, et al. Week 96 efficacy and safety of rilpivirine in treatment-naive, HIV-1 patients in two Phase III randomized trials. AIDS. 2013;27(6):939-950. doi:10.1097/ QAD.0b013e32835cee6e

50. Yoshinaga T, Seki T, Miki S, et al. Novel secondary mutations C56S and G149A confer resistance to HIV-1 integrase strand transfer inhibitors. Antiviral Res. 2018;152:1-9. doi:10.1016/j.antiviral.2018.01.013 
51. Smith SJ, Zhao XZ, Burke TR, Hughes SH. Efficacies of Cabotegravir and Bictegravir against drug-resistant HIV-1 integrase mutants. Retrovirology. 2018;15(1):37. doi:10.1186/s12977-018-0420-7

52. Oliveira M, Ibanescu RI, Anstett K, et al. Selective resistance profiles emerging in patient-derived clinical isolates with cabotegravir, bictegravir, dolutegravir, and elvitegravir. Retrovirology. 2018;15(1):1-14. doi:10.1186/s12977-017-0384-Z

53. Letendre S, Mills A, Hagins D, et al. Distribution in Cerebrospinal Fluid (CSF) of Cabotegravir (CAB) and Rilpivirine (RPV) after intramuscular administration of long-acting (LA) injectable suspensions in HIV-1-infected patients. In: HIV Glasgow. Glasgow; 2018: Abstract 0346. Available from: https://onlinelibrary.wiley.com/doi/ epdf/10.1002/jia2.25187

54. Baril J-G, Angel JB, Gill MJ, et al. Dual therapy treatment strategies for the management of patients infected with HIV: a systematic review of current evidence in ARV-naive or ARV-experienced, virologically suppressed patients. PLoS One. 2016;11(2):e0148231. doi:10.1371/journal.pone.0148231

55. Margolis AM, Heverling H, Pham PA, Stolbach A. A review of the toxicity of HIV medications. J Med Toxicol. 2014;10(1):26-39. doi:10.1007/s13181-013-0325-8

56. Orkin C, Llibre J, Gallien S, Antinori A, Behrens G, Carr A. Nucleoside reverse transcriptase inhibitor-reducing strategies in HIV treatment: assessing the evidence. HIV Med. 2018;19(1):1832. doi:10.1111/hiv.12534

57. Shaik JSB, Ford SL, Lou Y, et al. A phase 1 study to evaluate the pharmacokinetics and safety of cabotegravir in patients with hepatic impairment and healthy matched controls. Clin Pharmacol Drug Dev. Epub Feb 2019.

58. Bowers GD, Culp A, Reese MJ, et al. Disposition and metabolism of cabotegravir: a comparison of biotransformation and excretion between different species and routes of administration in humans. Xenobiotica. 2016;46(2):147-162. doi:10.3109/00498254.2015.1060372

59. Parasrampuria R, Ford SL, Lou Y, et al. A Phase I study to evaluate the pharmacokinetics and safety of cabotegravir in adults with severe renal impairment and healthy matched control participants. Clin Pharmacol Drug Dev. Epub Feb 2019.

60. Lou Y, Buchanan AM, Chen S, et al. Effect of cabotegravir on cardiac repolarization in healthy subjects. Clin Pharmacol Drug Dev. 2016;5(6):509-516. doi:10.1002/cpdd.272

61. Reese MJ, Bowers GD, Humphreys JE, et al. Drug interaction profile of the HIV integrase inhibitor cabotegravir: assessment from in vitro studies and a clinical investigation with midazolam. Xenobiotica. 2016;46(5):445-456. doi:10.3109/00498254.2015.1081993

62. Taskar KS, Patel A, Cozens SJ, et al. Prediction of renal OAT1 and OAT3 inhibition by cabotegravir using pbpk modelling. Conference on Retroviruses and Opportunistic Infections; 2019:Abstract 470. Seattle.

63. Ford SL, Sutton K, Lou Y, et al. Effect of rifampin on the single-dose pharmacokinetics of oral cabotegravir in healthy subjects. Antimicrob Agents Chemother. 2017;61:10. doi:10.1128/AAC.00487-17

64. Rajoli RKR, Curley P, Chiong J, et al. Predicting drug-drug interactions between rifampicin and long-acting cabotegravir and rilpivirine using physiologically based pharmacokinetic modeling. J Infect Dis. 2019;219(11):1735-1742. doi:10.1093/infdis/jiy726

65. Trezza C, Ford SL, Gould E, et al. Lack of effect of oral cabotegravir on the pharmacokinetics of a levonorgestrel/ethinyl oestradiol-containing oral contraceptive in healthy adult women. $\mathrm{Br} J$ Clin Pharmacol. 2017;83(7):1499-1505. doi:10.1111/bcp.13236

66. Blair C, Li S, Chau G, et al. Hormonal contraceptives do not alter cabotegravir PK in HIV-uninfected women HPTN 077. Conference on Retroviruses and Opportunistic Infections; 2019:Abstract 473. Seattle.

67. van der Galiën R, Ter Heine R, Greupink R, et al. Pharmacokinetics of HIV-integrase inhibitors during pregnancy: mechanisms, clinical implications and knowledge gaps. Clin Pharmacokinet. 2019;58 (3):309-323. doi:10.1007/s40262-018-0684-z
68. Watts DH, Stek A, Best BM, et al. Raltegravir pharmacokinetics during pregnancy. JAIDS J Acquir Immune Defic Syndr. 2014;67 (4):375-381. doi:10.1097/QAI.0000000000000318

69. Gilleece Y, Tariq S, Bamford A, et al. BHIVA Guidelines on the Management of HIV in Pregnancy and Postpartum. Hertfordshire, United Kingdom: BHIVA. Accessed https://www.bhiva.org/preg nancy-guidelines.

70. Office of AIDS Research Advisory Council. Recommendations for the Use of Antiretroviral Drugs in Pregnant Women with HIV Infection and Interventions to Reduce Perinatal HIV Transmission in the United States. Maryland, USA: AIDSinfo; 2019. Available from: https://aid sinfo.nih.gov/contentfiles/lvguidelines/PerinatalGL.pdf. Accessed April 30, 2019.

71. Mirochnick M, Shapiro DE, Morrison L, et al. Randomized trial of raltegravir-art vs efavirenz-art when initiated during pregnancy. Conference on Retroviruses and Opportunistic Infections; 2019: Abstract 39. Seattle.

72. Kintu K, Malaba T, Nakibuka J, et al. rct of dolutegravir vs efavirenzbased therapy initiated in late pregnancy: dolphin-2. Conference on Retroviruses and Opportunistic Infections; 2019. Seattle.

73. Rajoli RKR, Back DJ, Rannard S, et al. In silico dose prediction for long-acting rilpivirine and cabotegravir administration to children and adolescents. Clin Pharmacokinet. 2018;57(2):255-266. doi:10.1007/s40262-017-0557-x

74. National Institute of Allergy and Infectious Diseases. More Options for Children and Adolescents (MOCHA): oral and long-acting injectable cabotegravir and rilpivirine in HIV-Infected Children and Adolescents (MOCHA). Available from: https://clinicaltrials.gov/ ct2/show/NCT03497676?term=MOCHA+HIV\&rank=1. Accessed May 2, 2019.

75. Sherr L, Lampe F, Norwood S, et al. Adherence to antiretroviral treatment in patients with HIV in the UK: a study of complexity. AIDS Care. 2008;20(4):442-448. doi:10.1080/09540120701867032

76. Heestermans T, Browne JL, Aitken SC, Vervoort SC, KlipsteinGrobusch K. Determinants of adherence to antiretroviral therapy among HIV-positive adults in sub-Saharan Africa: a systematic review. BMJ Glob Heal. 2016;1(4):e000125. doi:10.1136/bmjgh2016-000125

77. Costa JDM, Torres TS, Coelho LE, Luz PM. Adherence to antiretroviral therapy for HIV/AIDS in Latin America and the Caribbean: systematic review and meta-analysis. J Int AIDS Soc. 2018;21(1): e25066. doi:10.1002/jia2.25066

78. Langebeek N, Gisolf EH, Reiss P, et al. Predictors and correlates of adherence to combination antiretroviral therapy (ART) for chronic HIV infection: a meta-analysis. BMC Med. 2014;12:142. doi:10.1186/ s12916-014-0141-2

79. Jackson A, Mcgowan I. Long-acting rilpivirine for HIV prevention. Curr Opin HIV AIDS. 2015;10(4):253-257. doi:10.1097/COH.0000 000000000160

80. ViiV Healthcare. Efficacy, safety and tolerability study of long-acting cabotegravir plus long-acting rilpivirine (CAB LA + RPV LA) in human-immunodeficiency virus-1 (HIV-1) infected adults. Available from: ClinicalTrials.gov. https://clinicaltrials.gov/ct2/show/ NCT03299049?term=Efficacy\%2C+Safety+and+Tolerability+Study + of + Long-acting + Cabotegravir + Plus + Long-acting + Rilpivirine $+\%$ $28 \mathrm{CAB}+\mathrm{LA}+\% 2 \mathrm{~B}+\mathrm{RPV}+\mathrm{LA} \% 29+\mathrm{in}+$ Human-immunodeficiency +Virus-1+\%28HIV-1\%29+Infected+Adults\&rank=1. Published 2018. Accessed April 30, 2019.

81. National Institute of Allergy and Infectious Diseases. The latitude Study: long-acting therapy to improve treatment success in daily life. ClinicalTrials.gov. Available from: https://clinicaltrials.gov/ct2/show/ NCT03635788?term=NCT03635788\&rank=1. Published 2019. Accessed April 30, 2019. 


\section{Publish your work in this journal}

HIV/AIDS - Research and Palliative Care is an international, peerreviewed open-access journal focusing on advances in research in HIV, its clinical progression and management options including antiviral treatment, palliative care and public healthcare policies to

control viral spread. The manuscript management system is completely online and includes a very quick and fair peer-review system, which is all easy to use. Visit http://www.dovepress.com/testimonials.php to read real quotes from published authors.

Submit your manuscript here: https://www.dovepress.com/hivaids—research-and-palliative-care-journa 